\title{
APTIDÃO FÍSICA DE CRIANÇAS E ADOLESCENTES ESCOLARES PRATICANTES DE ESPORTES EXTRACURRICULARES
}

\section{Physical fitness of schoolars children and adolescents practicers of extracurricular sports}

\author{
Rafael Abeche Generosi ${ }^{1,2}$, Bruno Manfredini Baroni', Ernesto Cesar Pinto Leal Junior ${ }^{1}$, \\ Gabriel Gustavo Bergmann², Daniel Carlos Garlipp², Marcelo Cardoso² \\ ${ }^{1}$ Laboratório do Movimento Humano (LMH/UCS). Instituto de Medicina do esporte (IME/UCS). \\ Universidade de Caxias do Sul (UCS). \\ ${ }^{2}$ Projeto Esporte Brasil (PROESP-BR/UFRGS). Laboratório de Pesquisa em Exercício (LAPEX/UFRGS). \\ Universidade Federal do Rio Grande do Sul (UFRGS).
}

\begin{abstract}
Resumo: Estudar os componentes relacionados à aptidão física e saúde é de fundamental importância para a compreensão destas variáveis nos diferentes contextos sociais, idades e gêneros sexuais. Este estudo propõe verificar a freqüência de ocorrência de crianças e adolescentes escolares nas zonas de classificação para a saúde, levando em consideração os critérios propostos pela literatura. Avaliou-se um total de 98 escolares (de nove à 17 anos), de ambos os sexos, praticantes de futebol/futsal/voleibol/natação, na cidade de Caxias do Sul/RS - Brasil. Para avaliar a aptidão física e a incidência de indivíduos nas zonas de classificação de saúde, foram utilizados os protocolos e critérios propostos pelo Projeto Esporte Brasil (PROESPBR, 2007). Nos resultados, encontrou-se um grande percentual de indivíduos classificados na zona Normal (IMC), e de Boa e Muito Boa saúde (demais componentes analisados). Conclui-se, desta forma, que há uma grande probabilidade da prática sistematizada, planejada e orientada de um esporte extracurricular ser o fenômeno responsável por elevar os níveis de aptidão física e saúde da grande maioria das crianças e adolescentes analisadas. Palavras-Chaves: Saúde, Esporte, Crianças, Adolescentes, Escola.
\end{abstract}

Abstract: To study the physical fitness and health components, it is fundamental to comprehend variables in different social contexts, ages and genders. The purpose of this study was to verify the frequency and occurrence of school children and adolescents in zones of health classification, considering the criteria proposed by literature. An amount of 98 scholars (nine to 17 years), of both sexes, practicers of soccer/futsal/volleyball/swimming, in Caxias do Sul/ RS - Brazil took part. The battery of tests and criteria proposed by Projeto Esporte Brasil (PROESP-BR, 2007) were used to analyze the physical fitness and incidence of individuals in zones of health classification. As a result, it was evidenced a big percentage of individuals classified in the Normal zone (BMI), and Good and Very Good zones of health (other components analyzed). It was concluded that there is a big probability of having a systematized, planned and oriented practice of an extracurricular sport as the responsible phenomenon for elevated levels of physical fitness and health in most of the analyzed children and adolescents.

Key words: Health, Sport, Children, Adolescents, School.

Aceito em 04/11/2008 - Rev. Educ. Fís. 2009 Mar: 144: 13-22. Rio de Janeiro - RJ - Brasil

\section{INTRODUÇÃO}

Diversas pesquisas são encontradas na literatura propondo como objetivo de estudo os problemas de saúde pública e de qualidade de vida das populações $(1,2,3,4,5)$. Há cinco décadas atrás as principais causas de mortalidade do ser humano eram devido às doenças infecto-contagiosas. À medida que a ciência e a tecnologia avançaram as principais causas de morte, tanto nos países desenvolvidos quanto nos em desenvolvimento, passaram a ser devido aos processos crônico-degenerativos como: doenças do coração, diabetes, câncer, obesidade ${ }^{(1,2)}$. Estas doenças mencionadas estão fortemente associadas à hipocinesia do sujeito, ou seja, à ausência ou pouca movimentação; o que é relatado na literatura como característico dos hábitos de vida de um ser humano sedentário ${ }^{(3,4)}$.

\section{É importante ressalvar que o sedentarismo não é} apenas identificado em indivíduos adultos e idosos. 
Esta característica tem se tornado cada vez mais peculiar às faixas etárias iniciais de vida (crianças e adolescentes). As crianças e adolescentes tem realizado menos atividade física em seu dia a dia. Atividades como assistir televisão, jogar vídeo game, "navegar" na internet, estão sendo costumeiras nestas idades ${ }^{(5,6,7)}$.

Como conseqüência deste processo de globalização, tem sido observado pela literatura que os níveis de aptidão física das crianças e adolescentes da atualidade são inferiores aos níveis de aptidão física dos seus respectivos pares há duas décadas atrás. No presente estudo, o termo aptidão física será definido como a expressão da capacidade funcional do indivíduo, direcionada à realização de esforços físicos associados à prática de atividade física, representada por um conjunto de componentes relacionados à saúde e ao desempenho atlético. ${ }^{(6)}$ Neste estudo, iremos referenciar apenas a aptidão física relacionada à saúde (ApFRS).

Dentre os componentes que constituem a ApFRS observam-se: a composição corporal (representada no presente estudo através do Índice de Massa Corporal $-\mathrm{IMC}=$ massa corporal/estatura ${ }^{2}$, a força/ resistência abdominal, flexibilidade e resistência cardiorrespiratória ${ }^{(8,9)}$. A força/resistência abdominal é comumente avaliada pelo teste de realização de repetições máximas abdominais durante o tempo de um minuto (sit up`s); a flexibilidade da região lombar e dos ísquios-tibiais é avaliada pelo teste de sentar e alcançar utilizando ou não como instrumento o Banco de Wells; e a resistência cardiorrespiratória que é avaliada por testes de campo ou por ergoespirometrias (modelo científico laboratorial) ${ }^{(9)}$.

Estudar os componentes relacionados à aptidão física e saúde é de fundamental importância para a compreensão destas variáveis nos diferentes contextos sociais, em diferentes faixas etárias da vida e gêneros. Ainda, é considerado importante, além de descrever esta situação da população estudada frente a estas variáveis, comparar os resultados encontrados frente aos critérios já propostos na literatura. Ou seja, avaliar e analisar o nível de aptidão física do grupo estudado a partir de pontos de corte que estabeleçam alguma relação com a possibilidade de risco de surgimento de doenças hipocinéticas.

Desse modo, o presente estudo propõe verificar a freqüência de ocorrência de crianças e adolescentes nas zonas de classificação de saúde, levando em consideração os critérios relacionados à saúde propostos pela literatura do PROESP-BR ${ }^{(9)}$.

\section{METODOLOGIA}

O delineamento metodológico do presente estudo configura-se como descritivo comparativo, de acordo com as proposições de Thomas, Nelson e Silverman (10) e Gaya et al. (11). Trata-se de uma investigação sobre os níveis de aptidão física e saúde de um grupo de crianças e adolescentes escolares e dos percentuais de ocorrências de indivíduos nas zonas de classificação de saúde a partir das normas nacionais de avaliação da aptidão física.

\section{Amostra}

No estudo foi avaliado um total de 98 crianças e adolescentes escolares da cidade de Caxias do Sul/ RS - Brasil, com faixa etária entre nove e dezessete anos, de ambos os sexos. Na TABELA 1, a seguir, é retratada a distribuição do número de sujeitos avaliados conforme a idade e o sexo. 
15 Rev. Educ. Fís. 2009 Mar: 144: 13-22. Rio de Janeiro - RJ - Brasil

TABELA 1.

DISTRIBUIÇÃO DA AMOSTRA TOTAL POR IDADES E SEXOS.

\begin{tabular}{cccc}
\hline Idades & Masculino & Feminino & Total \\
\hline 9 anos & 2 & 0 & 2 \\
10 anos & 5 & 2 & 7 \\
11 anos & 12 & 1 & 13 \\
12 anos & 20 & 2 & 22 \\
13 anos & 14 & 10 & 24 \\
14 anos & 6 & 4 & 10 \\
15 anos & 5 & 1 & 6 \\
16 anos & 11 & 1 & 12 \\
17 anos & 2 & 0 & 2 \\
\hline Total & 77 & $\mathbf{2 1}$ & $\mathbf{9 8}$ \\
\hline
\end{tabular}

No grupo dos meninos $(n=77)$ destaca-se que a idade média encontrada foi de $12,87 \pm 1,98$ anos e no grupo das meninas $(n=21)$ foi de $12,95 \pm 1,43$ anos. Dentre os meninos, 42 são praticantes de futebol, 12 são praticantes de futsal e 23 de natação. Já no sexo feminino, 12 são praticantes de voleibol e 9 de natação.

Esta amostra foi escolhida de forma intencional, devido à acessibilidade dos grupos. Ressalvase que estas crianças e adolescentes avaliados são integrantes das escolinhas desportivas da Universidade de Caxias do Sul, Projeto UCS Olimpíadas. Como o Instituto de Medicina do Esporte, que foi o instituto responsável pela aplicação da bateria de testes, está vinculado a UCS; houve uma predisposição na seleção da amostra.

Como critérios de inclusão da amostra foram destacados os seguintes itens:

- Todos os sujeitos deveriam estar matriculados em uma escola da rede pública de ensino da cidade de Caxias do Sul, Rio Grande do Sul, Brasil;
- Possuir freqüência regular na escola em um dos turnos do dia;

- Na grade curricular da escola deveria ter o planejamento de três aulas de Educação Física Escolar (EFE) por semana;

- O sujeito deveria ter uma freqüência mínima de $75 \%$ das aulas de (EFE).

- Os sujeitos deveriam integrar um programa de treinamento esportivo (futebol, futsal, voleibol ou natação) do Projeto UCS Olimpíadas, de forma extracurricular.

- Estes treinamentos (futebol, futsal, voleibol ou natação) ocorrem com uma freqüência mínima semanal de três vezes sendo a duração média da sessão de treinamento é de uma hora e meia;

- A freqüência de participação dos treinamentos deve ser de $90 \%$.

- A experiência de prática da modalidade deve ser superior a um ano;

Previamente as coletas de dados, os responsáveis pelos sujeitos avaliados tomaram conhecimento do 
estudo e assinaram um termo de consentimento livre e esclarecido, conforme determina a resolução 196/96 do Conselho Nacional de Saúde (CNS). O estudo foi submetido e aprovado pelo Comitê de Ética em Pesquisa da Universidade do Vale do Paraíba (UNIVAP).

\section{Instrumentos e Procedimentos de Coleta}

Na TABELA 2 são retratadas as variáveis analisadas neste trabalho, os seus respectivos testes, protocolos e instrumentos. Estes testes são sugeridos pelo Projeto Esporte Brasil (PROESP-BR, 2007) ${ }^{(9)}$.

\section{Critérios de Referência}

Para comparar a aptidão física relacionada à saúde e determinar os percentuais de freqüência de ocorrência de indivíduos nas zonas de classificação de saúde os indivíduos foram classificados de acordo com os critérios de zona saudável de aptidão física. Utilizaram-se para o Índice de Massa Corporal (IMC) os critérios propostos por Conde e Monteiro ${ }^{(12)} \mathrm{e}$ para a força/resistência abdominal, flexibilidade e resistência cardiorrespiratória, os critérios do Projeto Esporte Brasil (9). Ressalva-se que o PROESP-BR (9) também adota para o IMC os critérios propostos por Conde e Monteiro ${ }^{(12)}$.

De acordo com os critérios de Conde e Monteiro ${ }^{(12)}$ as crianças e jovens brasileiros são classificados entre quatro categorias conforme o IMC: Baixo Peso, Normal, Excesso de Peso e Obesidade. O PROESPBR (9) adota um sistema referenciado em normas. Tendo como referência os padrões da população brasileira estratificada por idade (sete a 17 anos) e sexo (masculino e feminino), definem-se seis categorias de aptidão física conforme é apresentado na TABELA 3.

TABELA 2.

VARIÁVEIS, TESTES, PROTOCOLOS E INSTRUMENTOS DA COLETA DE DADOS DO PRESENTE ESTUDO.

Variável

Teste
Protocolo

\section{Instrumento}

Índice de Massa

Corporal (IMC) - Massa

Corporal e Estatura

Massa corporal (MC) e estatura (EST)

Abdominal em 1 minuto

Abdominal (sit up's)

Flexibilidade

(sit and reach)

Resistência

Cardiorrespiratória

Sentar e Alcançar (SA)
Verificar qual a $\mathrm{MC}(\mathrm{Kg})$

e a EST $(m)$ do sujeito.

Após, divide-se a MC pela

$\mathrm{EST}^{2}$ e tem-se o IMC

Avaliar o maior número

de repetições de flexões

abdominais dentro do

período de 1 minuto

Avaliar o índice de

flexibilidade em um

movimento de SA

Avaliar a maior distância

percorrida (metros) dentro

do período de $9 \mathrm{~min}$
Balança com precisão de $100 \mathrm{~g}$ e estadiômetro com precisão de $1 \mathrm{~mm}$.

\section{Colchonete e} cronômetro

Banco de Wells

Cronômetro 
TABELA 3.

NORMAS E CATEGORIAS PARA AVALIAÇÃO DA APTIDÃO FÍSICA.

Valores em Percentil

Valores inferiores ao percentil 20

Valores entre o percentil 20 e 40

Valores entre o percentil 40 e 60

Valores entre o percentil 60 e 80

Valores entre o percentil 80 e 98

Valores iguais ou superiores ao percentil 98
Categorias de Aptidão Física

Muito Fraco (risco à saúde)

Fraco

Razoável

Bom

Muito Bom

Excelente

\section{Tratamento Estatístico}

No tratamento dos dados inicialmente foi realizado um estudo exploratório a fim de avaliar a normalidade destes seguindo os pressupostos da análise paramétrica. Esta análise constou da inspeção da simetria (Skewness), do achatamento (Kurtosis) e do teste de normalidade Shapiro-Wilk. Como os dados demonstratam normalidade, seguiu-se com a as análises descritivas usuais de média e desvio padrão. Após, determinou-se o percentual de freqüência de ocorrência de indivíduos distribuídos nas zonas de classificação relacionadas à aptidão física e saúde propostas pela literatura. Todos os dados foram tratados no pacote estatístico SPSS for Windows, versão 13.0.

\section{RESULTADOS}

Na FIGURA 1, são retratados os percentuais de freqüência de ocorrência dos sujeitos avaliados no presente estudo conforme as zonas de classificação para IMC. Quanto ao sexo feminino, pode ser observado que nenhuma das meninas avaliadas foi classificada nas categorias de baixo peso e obesidade. Já no sexo masculino os percentuais para essas categorias foram de $12,99 \%$ e $2,6 \%$, respectivamente. Por outro lado, a grande maioria dos indivíduos avaliados, meninos $(70,13 \%)$ e meninas $(80,95 \%)$, classificaram-se dentro da zona Normal para o IMC.

FIGURA 1.

PERCENTUAL DE FREQÜÊNCIA DE OCORRÊNCIA NAS ZONAS DE CLASSIFICAÇÃO DOS CRITÉRIOS DE SAÚDE PARA IMC.

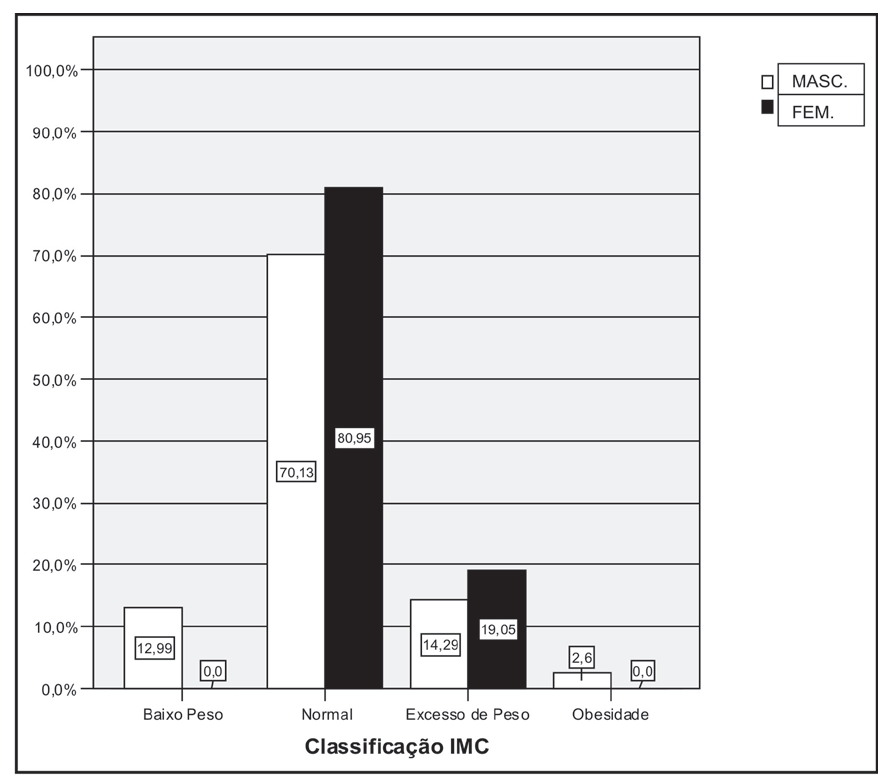


Na FIGURA 2, são apresentados os percentuais de freqüência de ocorrência dos sujeitos avaliados conforme as zonas de classificação para força/ resistência abdominal. Como pode ser observado, nenhuma das meninas avaliadas foi classificada nas categorias Muito Fraco e Fraco. A maior parte dos indivíduos avaliados, meninos $(79,22 \%)$ e meninas $(90,84 \%)$ classificou-se acima da Zona de Boa saúde, ou seja, acima do percentil 60. Ressalvase que neste componente de saúde, encontrou-se $3,9 \%$ (meninos) e 4,76\% (meninas) com o percentil igual ou maior a 98, ou seja, classificados na zona de Excelência.

FIGURA 2.

PERCENTUAL DE FREQÜÊNCIA DE OCORRÊNCIA NAS ZONAS DE CLASSIFICAÇÃO DOS CRITÉRIOS DE SAÚDE PARA FORÇA/ RESISTÊNCIA ABDOMINAL.

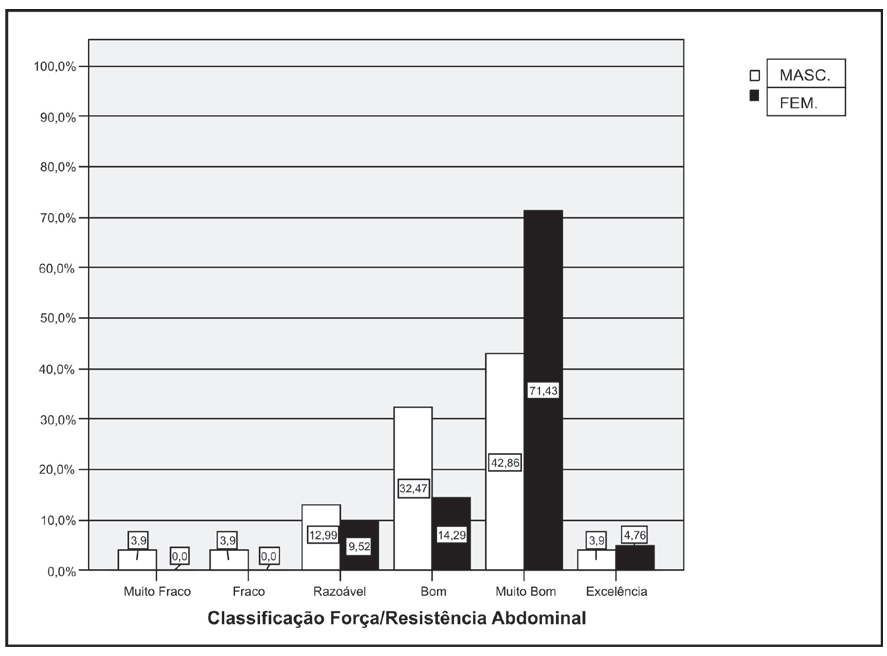

Na FIGURA 3, são apresentados os percentuais de freqüência de ocorrência dos sujeitos avaliados conforme as zonas de classificação para flexibilidade. Observa-se que nenhuma menina avaliada foi classificada nas categorias Muito Fraco e Excelência. A grande maioria dos indivíduos avaliados, meninos $(72,64 \%)$ e meninas $(90,47 \%)$ classificou-se acima da Zona Razoável de saúde, ou seja, acima do percentil 40. Ressalva-se que para os meninos, encontrou-se uma freqüência de ocorrência mais próxima à esperada nas zonas de classificação de saúde para o componente de flexibilidade.

\section{FIGURA 3.}

PERCENTUAL DE FREQÜÊNCIA DE OCORRÊNCIA NAS ZONAS DE CLASSIFICAÇÃO DOS CRITÉRIOS DE SAÚDE PARA FLEXIBILIDADE.

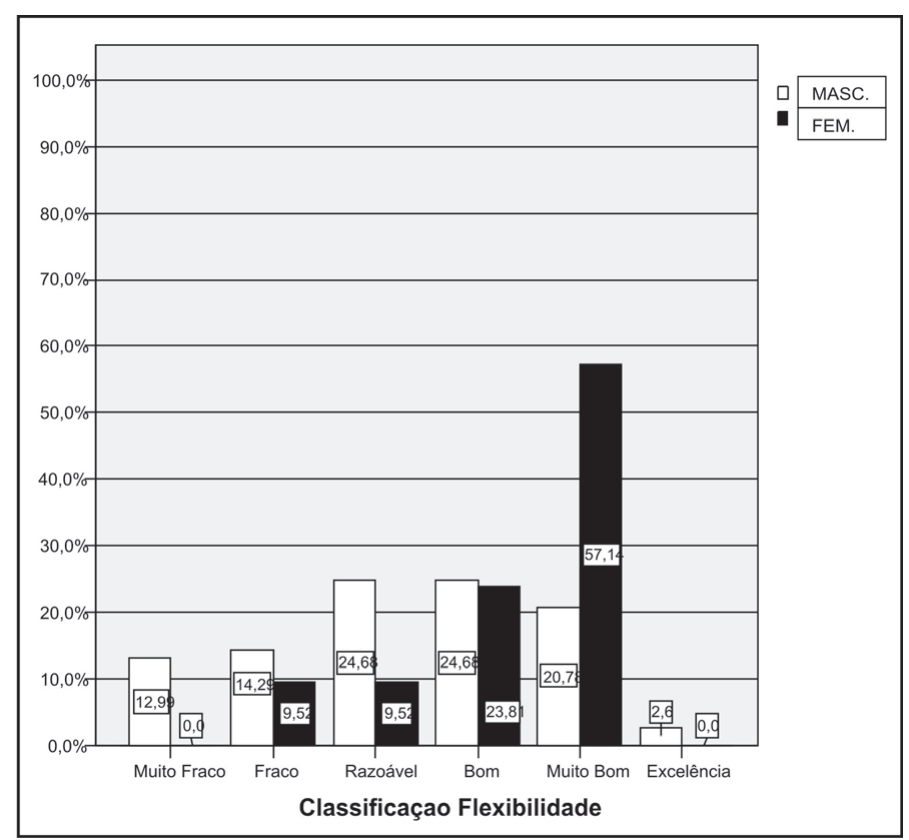

Na FIGURA 4, são apresentados os percentuais de freqüência de ocorrência dos sujeitos avaliados conforme as zonas de classificação para resistência cardiorrespiratória. Assim como observado ocorrido na flexibilidade, nenhuma menina avaliada foi classificada nas categorias Muito Fraco e Fraco. A grande maioria dos indivíduos avaliados, meninos $(75,33 \%)$ e meninas $(95,24 \%)$ classificou-se na Zona de Boa saúde ou acima da Zona de Boa saúde, ou seja, acima do percentil 60. Ressalvase que neste componente de saúde encontrou-se $2,6 \%$ (meninos) e $4,76 \%$ (meninas) com o percentil igual ou maior a 98, ou seja, classificados na zona de Excelência. 
FIGURA 4.

PERCENTUAL DE FREQÜÊNCIA DE OCORRÊNCIA NAS ZONAS DE CLASSIFICAÇÃO DOS CRITÉRIOS DE SAÚDE PARA RESISTÊNCIA

\section{CARDIORRESPIRATÓRIA.}

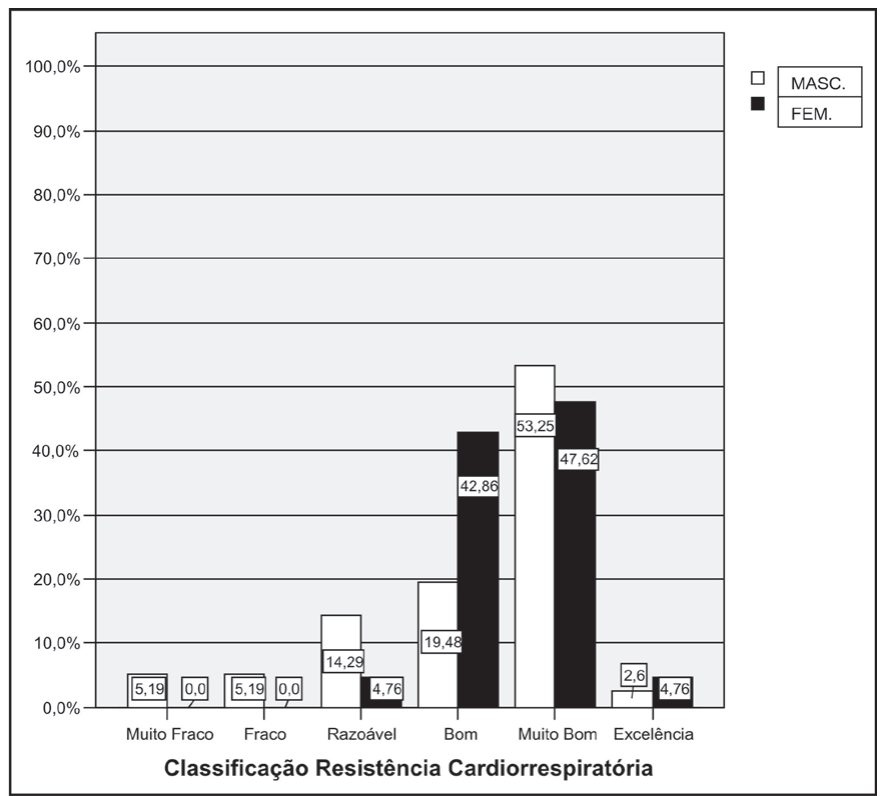

\section{DISCUSSÃO}

Quanto ao índice de Massa Corporal, podese observar que $70,13 \%$ dos meninos e $80,95 \%$ das meninas avaliadas classificaram-se na zona Normal. Estes valores percentuais são bastante satisfatórios tendo em vista que uma pequena parcela da população avaliada $(29,87 \%$ - meninos e $19,05 \%$ - meninas) ficou classificada fora das zonas satisfatórias para o IMC.

Eliakin et al. ${ }^{(13)}$, analisaram os efeitos de um programa de treinamento de exercícios físicos, com duração de três a seis meses, no componente IMC de uma população de 177 crianças obesas, com idades variando de 6 a 16 anos). Neste estudo foi utilizado como grupo controle, 25 crianças obesas de mesma faixa etária, que não iriam praticar os exercícios físicos propostos. O grupo de sujeitos que realizou o treinamento obteve uma significante $(p<0,05)$ redução nos valores de IMC, ao passo que o grupo controle obteve um incremento nos valores.

Desta forma, faz-se menção o fato das crianças e adolescentes avaliadas no presente estudo realizarem uma prática desportiva extracurricular planejada, sistematizada e orientada, aumentando a probabilidade do sujeito ser classificado na zona satisfatória de IMC. Diferentemente de uma outra criança ou adolescente, que não possui a mesma prática de exercícios físicos, ou que possui hábitos característicos de um estilo de vida sedentário; predispondo a sua classificação de IMC nas zonas de excesso de peso e obesidade.

Ressalva-se que a decisão sobre qual critério adotar para a classificação do IMC é um tema que continua em discussão na literatura. Recentemente, existe uma corrente metodológica firmando a opção pelo conjunto de curvas que retroagem os valores da classificação dos adultos jovens para os indivíduos com idade abaixo de 18 ou 20 anos (método LMS) ${ }^{(12)}$.

No componente de força/resistência abdominal observou-se que $79,22 \%$ dos meninos e $90,84 \%$ das meninas foram classificados dentro e/ou acima da zona de boa saúde, ou seja, acima do percentil 60 . Além disso, nenhuma menina foi classificada abaixo do percentil 40 e apenas $7,8 \%$ dos meninos foram classificados nas zonas Muito Fraco e Fraco.

Para Glaner (14), a prática regular de exercício físico influencia positivamente o desenvolvimento da capacidade de força/resistência abdominal. Já, no estudo desenvolvido por Seabra, Maia e Garganta (15), onde foram avaliados o crescimento, a maturação e a aptidão física de jovens futebolistas e não futebolistas de Portugal, foi identificado que os futebolistas obtiveram resultados mais expressivos do que seus respectivos pares, não futebolistas, na variável de força/resistência abdominal.

Alguns autores observam que as crianças e adolescentes que participam de programas de treinamento que desenvolvem a capacidade de resistência cardiorrespiratória e força muscular (ex.: prática desportiva) tem uma maior probabilidade de terem um incremento na sua produção hormonal de testosterona. Este processo fisiológico favoreceria ao sujeito o desenvolvimento da sua massa muscular ${ }^{(16)}$, aumentando assim a condição para que a criança ou 
adolescente realize um maior número de repetições abdominais no sit up's test.

Para o terceiro componente da aptidão física relacionada à saúde avaliado no presente estudo, flexibilidade (FIGURA 3), foi encontrado para os meninos uma freqüência de ocorrência de 24,68\% na categoria Bom, 20,78\% na categoria Muito Bom e 2,6\% na Excelência. Para as meninas, encontrouse um grande contingente da amostra $(80,95 \%$ de freqüência de ocorrência) dentro das zonas de boa e/ou muito boa saúde.

Para Maffulli (17), é incerto o fato de que a flexibilidade possa ser influenciada pelas particularidades anatômicas, fisiológicas, genéticas ou ambientais, dentre elas a pratica habitual de exercício físico. No entanto, sabe-se que quanto maior forem os estímulos da capacidade de flexibilidade durante um programa de treinamento sistematizado, planejado e orientado, maior será o seu desenvolvimento ${ }^{(18,19)}$.

O componente de flexibilidade é uma capacidade altamente específica. Isto que dizer que o sujeito pode ter uma boa amplitude de movimento no sit and reach test e uma menor amplitude nos movimentos da cintura escapular (ex: flexão e extensão de ombro). Ressalva-se que nenhuma medida por si só é representativa da flexibilidade global do sujeito. É necessária a aplicação de vários testes, que incidam sobre as diferentes estruturas articulares, para ser observado de forma mais consistente o componente em destaque (20). O desempenho no teste sit and reach, propriamente dito, tem forte associação com incidência de dores na região lombar ${ }^{(21)}$.

Quando analisado a componente resistência cardiorrespiratória foram encontrados $75,33 \%$ dos meninos e $95,24 \%$ das meninas classificados acima do percentil 60 . Ainda, 2,6\% dos meninos e 4,76\% das meninas classificaram-se na zona de Excelência, ou seja, igual ou acima do percentil 98.

As alterações cardiovasculares decorrentes dos exercícios aeróbios são amplamente relatadas na literatura, sendo as principais: aumento do volume do coração, do volume de ejeção, da concentração de eritrócitos e hemoglobina no sangue, diminuição da freqüência cardíaca de repouso e da pressão arterial, melhoria na vascularização periférica ${ }^{(22)}$. Desta forma, indivíduos submetidos a um programa de treinamento desportivo de forma sistematizada e orientada, tem uma maior predisposição a apresentarem resultados considerados satisfatórios para a saúde no componente resistência cardiorrespiratória.

Em um estudo onde se compararam variáveis de aptidão física relacionadas à saúde (ApFRS) em adolescentes que participam somente das aulas de Educação Física curricular, com adolescentes que participam adicionalmente de programas extracurriculares de treinamento desportivo, observouse que o segundo grupo de indivíduos apresentou melhores performances nos testes de ApFRS. Ainda, no componente de resistência cardiorrespiratória encontrou-se a maior diferença de desempenho ${ }^{(23)}$.

Resultados positivos no desenvolvimento da função cardiorrespiratória foram encontrados em adolescentes caracterizados como obesos e que foram submetidos a uma intervenção de prática de exercício físico ${ }^{(24)}$. Este estudo ainda reporta que a prevenção das doenças cardiovasculares deve ter início na infância, tendo em vista que os fatores de risco já estão presentes nas fases iniciais da vida.

É pertinente ressaltar a importância da prática do exercício físico e esporte de forma sistematizada, com programas de treinamento estruturados, acompanhados e orientados por profissionais capacitados. Assim, os objetivos do treinamento tornam-se possíveis de serem alcançados e, conseqüentemente, influenciam de forma positiva no crescimento e desenvolvimento do adolescente. Desta forma, há uma maior probabilidade da promoção da saúde, socialização, autoconhecimento corporal, melhora da auto-estima e prevenção da obesidade e outras doenças crônico-degenerativas.

Por fim, salienta-se que novos estudos sejam realizados avaliando populações de outras regiões do Brasil, em outros contextos sócio-culturais, incluindo novas variáveis nas análises como o perfil sócio- 
Rev. Educ. Fís. 2009 Mar: 144: 13-22. Rio de Janeiro - RJ - Brasil

econômico da família, perfil nutricional do sujeito avaliado, hábitos de vida e maturação. Aspectos que no presente estudo não foram abordados. Estas novas pesquisas podem otimizar o conhecimento em torno dos níveis ideais de aptidão física e saúde das crianças e adolescentes brasileiros, nas diferentes faixas etárias e sexo, bem como nos pontos de corte recomendados pela literatura e as zonas de classificação de saúde.

\section{CONCLUSÃO}

Através dos resultados obtidos e das análises realizadas conclui-se que há uma grande probabilidade da prática sistematizada, planejada e orientada de uma modalidade desportiva extracurricular, ou seja, fora do ambiente escolar, ter sido o fenômeno responsável por elevar os níveis de aptidão física e saúde da grande maioria das crianças e adolescentes avaliadas no presente estudo. Em todas as variáveis mensuradas (IMC, força/resistência abdominal, flexibilidade e resistência cardiorrespiratória) houve um grande percentual de freqüência de ocorrência de indivíduos classificados na zona Normal (IMC), e de Boa e Muito Boa saúde (demais componentes). A promoção da saúde e a prevenção das doenças cardiovasculares devem ter início ainda na infância e adolescência, tendo em vista que os fatores de risco já estão presentes nas fases iniciais da vida.

\section{REFERÊNCIAS BIBLIOGRÁFICAS}

1. Katzmarzyk PT, Srinivasan SR, Chen W, Malina RM, Bouchard C, Berenson G. Body mass index, waist circumference and clustering of cardiovascular risk factors in a biracial sample of children and adolescents. Pediatr 2004;114:198-205.

2. Reed KE, Warburton DER, Mckay HA. Determining Cardiovascular disease Risk in Elementary School Children: Developing a healthy heart score. J Sports Sci Med 2007;6:142-148.
3. Ribeiro RQC, Lotufo PA, Lamounier JA, Oliveira RG, Soares JF, Botter DA. Fatores Adicionais de Risco Cardiovascular Associados ao Excesso de Peso em Crianças e Adolescentes: O estudo do coração de Belo Horizonte. Arq Bras Cardiol 2006;86(6):408-418.

4. Rodriques AN, Perez AJ, Carletti L, Bissoli NS, Abreu GR. Aptidão Cardiorrespiratória e Associações com Fatores de Risco Cardiovascular em Adolescentes. J Pediatr 2007;83(5):429-435.

5. Bergmann MLA, Halpern R, Bergmann GG. Perfil lipídico, de aptidão cardiorrespiratória, e de composição corporal de uma amostra de escolares de $8^{\mathrm{a}}$ série de Canoas/RS. Rev Bras Med Esp 2008;14:22-27.

6. Caspersen CJ, Pereira AM, Curran KM. Changes in physical activity and patterns in the United States, by sex and cross-sectional age. Med Sci Sports Exerc 2000;32(9):1601-1609.

7. Bergmann GG, Araújo MLB, Garlipp DC, Lorenzi TDC, Gaya A. Alteração anual no crescimento e na aptidão física relacionada à saúde de escolares. Rev Bras Cineantropom Desempenho Hum 2005;7(2):55-61.

8. Gaya A, Guedes DPG, Torres L, Cardoso M, Poletto A, Silva M. Aptidão Física Relacionada à Saúde: um estudo piloto sobre o perfil de escolares de 7 a 17 anos da Região Sul do Brasil. Revista Perfil 2002;6(6):50-60.

9. Projeto Esporte Brasil: Observatório permanente dos indicadores de saúde e fatores de prestação esportiva em crianças e jovens. Manual de Aplicação de Medidas e Testes, Normas e Critérios de Avaliação. Gaya ACA, Silva GMG. Disponível em: <http://www.proesp.ufrgs.br>Acesso em: 07 Ago 2007.

10. Thomas JR, Nelson JK, Silverman SJ. Métodos de Pesquisa em Educação Física. $5^{\mathrm{a}}$. ed. Porto Alegre: Artmed, 2007.

11. Gaya ACA. Ciência do Movimento Humano. Introdução à Metodologia de Pesquisa. Porto Alegre: Artmed, 2008.

12. Conde WL, Monteiro CA. Body mass index cutoff points for evaluation of nutritional status in Brazilian children and adolescents. J Pedriatr 2006;82(4):266-272.

13. Eliakim A, Kaven G, Berger I, Friedland O, Wolach B, Nemet D. The effect of a combined intervention on body mass index and fitness in obese children and adolescents. A clinical experience. Eur J Pediatr 2002;161:449-454. 
14. Glaner MF. Crescimento e aptidão física relacionada à saúde em adolescentes rurais e urbanos em relação a critérios de referência. Revista Brasileira de Educação Física e Esportes 2005;19(1):13-24.

15. Seabra A, Maia JA, Garganta R. Crescimento, maturação, aptidão física, força explosiva e habilidades motoras específicas. Estudo em jovens futebolistas e não futebolistas do sexo masculino dos 12 aos 16 anos. Revista Portuguesa de Ciências do Desporto 2001;1(2):22-35.

16. Faigenbaum A. Strenght training and children's health. JOPERD 2001;72:24-30.

17. Maffulli N. Children in sports. The European prospective. In: Chan KM, Micheli LJ. Journal Sports and children. Honk Kong: Williams \& Asia-Pacific, p.97-107, 1998.

18. Guedes DP, Guedes JERP. Influência da prática da atividade física em crianças e adolescentes: uma abordagem morfológica e funcional. Revista APEF Londrina 1995;10(17):3-25.

19. Baltaci G, Un N, Tunay V, Besler A, Gerceker S. Comparison of three different sit and reach tests for measurement of hamstring flexibility in female university students. Br J Sports Med 2003;37(1):59-61.

20. Da Silva DJL, Dos Santos JAR, De Oliveira BMPM. A flexibilidade em adolescentes. Um contributo para a avaliação global. Rev Bras Cineantropom Desempenho Hum 2006;8(1):72-79.

21. Sjolie AN. Low-back pain in adolescents is associated with poor hip mobility and high body mass index. Scan $\mathrm{J}$ Med Sci Sports 2004;14:168-175.

22. Sasaki JE, Dos Santos MG. O papel do exercício aeróbico sobre a função endotelial e sobre os fatores de risco cardiovasculares. Arq Bras Cardiol 2006;87:e227e233.

23. Koutedakis Y, Bouziotas C. National physical education curriculum. Motor and cardiovascular health related fitness in Greek adolescents. Br J Sports Med 2003;37:311-314.

24. Watts K, Beye P, Siafarikas A, Davis EA, Jones TW O'driscoll G, Green DJ. Exercise training normalizes vascular dysfunction and improves central adiposity in obese adolescents. J Am Coll Cardiol 2004;43(10):18231827.

\section{Endereço:}

Universidade de Caxias do Sul Instituto de Medicina do Esporte

Rua Francisco Getúlio Vargas, 1130 - Bloco 70 Bairro Petrópolis

CEP: 95020-972

Telefone: (54) 3218-2736 / Celular: (54) 91765519

Rafael Abeche Generosi - rafaelgenerosi@hotmail.com Bruno Manfredini Baroni - baroni09@yahoo.com.br Ernesto Cesar Pinto Leal Junior - ecplealj@ucs.br Gabriel Gustavo Bergmann - gabrielgbergmann@yahoo. com.br Daniel Carlos Garlipp - dcgarlipp@gmail.com Marcelo Cardoso - marcelocardoso390@hotmail.com 